\title{
MUSCULAR DYSTROPHIES; NO MORE A RARITY \\ drnadiaf@hotmail.com
}

1. Assistant Professor

Department of Morbid Anatomy and Histopathology University of Health Sciences, Lahore

2. Assistant Professor Department of Human Genetic, University of Health Sciences, Lahore

3. Orthopedic Surgeon

Pakistan Society of

Rehabilitation of Disabled, Lahore

4. Assistant Professor

Department of Orthopedic Surgery, Children Hospital, Lahore

5. Research Associate University of Health Sciences, Lahore

6. Biostatistician

University of Health Sciences, Lahore

7. Professor of Pathology, University of Health Sciences, Lahore

Correspondence Address:

Dr. Nadia Naseem

Assistant Professor

Department of Morbid Anatomy and Histopathology

University of Health Sciences Lahore Pakistan

drnadiaf@hotmail.com

Article received on: $11 / 03 / 2016$

Accepted for publication:

28/07/2016

Received after proof reading:

10/09/2016

\section{Dr. Nadia Naseem, ${ }^{1}$ Dr. Saqib Mahmood, ${ }^{2}$ Dr. Afzal Hussain, ${ }^{3}$ Dr. Mumtaz Hussain, ${ }^{4}$ Miss Ayesha Munir, ${ }^{5}$ Mr. Waqas Latif, ${ }^{6}$ Dr. A.H. Nagi ${ }^{7}$}

ABSTRACT... Objectives: Muscular dystrophies are classified into different types based on their age of onset, clinical severity, rate of progression, distribution of muscles weakness, pattern of inheritance and the genes involved. As muscular dystrophies are relatively uncommon disorders, very little work has been done in Pakistan. This study has classified (for the first time in Pakistan) the patients with different types of muscular dystrophies by clinical and biochemical correlation. Study Design: Observational, descriptive study. Setting: Departments of Morbid Anatomy and Histopathology and Human Genetics and Molecular Biology at University of Health Sciences Lahore. Period: Three years' (March, 2012-March 2015). Methodology: A total of 100 patients comprising of all age groups and both genders and with strong clinical suspicion of muscular dystrophy, with or without positive family history, were included. Detailed clinical history and physical examination findings were recorded followed by estimation of serum Creatine Phosphokinase (CPK), Lactate Dehyrogenase $(\mathrm{LDH})$ and Aldolase levels. Results: A total of 77 males $(\mathrm{M})$ and 23 females $(\mathrm{F})$ were included. Mean age of the patients was $12.1990 \pm 6.69913$ years. Parents of 76 patients reported with consanguineous marriages while positive family history was reported in 31 patients. Eighty two cases managed to ambulate with support of other persons while $n=14$ patients were totally non ambulatory. Symmetrical muscle involvement (lower limb followed by upper limb) was shown by 87 cases (including all males and $n=22$ females). Involvement of the lower limbs as a primary weakness followed by symmetrical and severe involvement of the upper limb girdle and lower limb girdle suggested limb girdle muscle dystrophy (LGMD) in 9 (6F,3M) patients. Involvement of shoulders and upper limbs as a primary weakness followed by facial muscles and infrequent involvement of lower limbs suggested fascioscapulohumeral muscle dystrophy (FSHMD) in 2 cases. Involvement of upper limbs as a primary weakness followed by lower limbs with a moderate rise in CPK levels and mild phenotype was seen in two female patient which was quite suggestive of dysferlinopathy. Only two male patients reported onset of weakness and ambulation loss before 1 year of age that may represent congenital muscle dystrophy (CMD), dystroglycanopathy or congenital onset of LGMD (CLGMD). Mean serum $\mathrm{CPK}, \mathrm{LDH}$ and Aldolase levels were $2376.6364 \pm 910.78963 \mathrm{U} / \mathrm{L}, 1030.7800 \pm 180.1620 \mathrm{U} / \mathrm{L}$ and $10.089 \pm 1.525 \mathrm{U} / \mathrm{L}$ respectively that demonstrated significant association $(p<0.05)$ with characteristic clinical features. Conclusion: Muscular dystrophies are not only restricted to dystrophinopathies in our population. Apart from clinical and biochemical parameters, appropriate histological and/or gene mutation analysis is mandatory for precise classification of these disorders.

Key words: Duchenne muscle dystrophy (DMD), Becker's muscle dystrophy (BMD), Limb girdle muscular dystrophy (LGMD), Fascioscapulohumeral muscular dystrophy (FSHMD), Congenital muscle dystrophy (CMD)

Article Citation: Naseem N, Mahmood S, Hussain A, Hussain M, Munir A, Latif W, Nagi AH. Muscular dystrophies; no more a rarity. Professional Med J 2016;23(9):11101117. DOI: $10.17957 / \mathrm{TPMJ} / 16.3344$

\section{INTRODUCTION}

Muscular dystrophies are classified into different types based on their age of onset, clinical severity, rate of progression, distribution of muscles weakness, pattern of inheritance and the genes involved. ${ }^{1}$ The original classification scheme proposed by Walton and Nattrass $(1954)^{2}$ included three major groups of muscular dystrophies i-e Duchenne type (Duchenne and Becker), Limbgirdle type, and Fascioscapulohumeral type and 
there were three comparatively uncommon forms i-e Distal Occulopharyngeal and Congenital type of muscular dystrophies. A significant number of muscle dystrophies are caused by the mutations that affect the normal assembly of the dystrophin and the dystrophin associated glycoprotein complex (DAGC) at the sarcolemma. ${ }^{3,4}$ Diagnosis of a muscular dystrophy usually begins with the clinical suspicion and it has proven to have a very high positive predictive value. On the other hand, the serum creatine phosphokinase (CPK) level is now the single most important blood value to be obtained when myopathy is being considered. Creatine phosphokinase is the enzyme that catalyses the reversible reaction of phosphocreatine and ADP conversion into creatine and ATP and thus involved in the generation of ATP in the muscle cells. Serum CPK is found to be raised in majority of the carriers, as well as in patients, even well before the symptoms appear. Furthermore this might be of help in cases with inconclusive findings on EMG. The increased serum CPK levels in DMD have been attributed to increased creatine kinase released from skeletal muscle fibers judging from the isoform patterns of the enzyme. ${ }^{5}$ The increase of CPK is speculated to result from leakage of the enzyme from diseased muscle fibers. ${ }^{6}$ The measurement of CPK is an easy, quick, most common, noninvasive test and considered best among the first line investigations for the detection of carriers. ${ }^{7}$ Serum aldolase level further helps in suggesting myopathy, because of its longer half-life in serum and it might be raised in setting of myopathy with normal CPK level. ${ }^{4,8}$ Aldolase catalyzes the reversible splitting of fructose $1: 6$-diphosphateto glyceraldehyde-3-phosphate and dihydroxyacetone phosphate. Diagnostically it is of value in distinguishing primary muscular disease from neurogenic muscular atrophy. The determination is of value in the diagnosis of muscular dystrophy, and in the detection of this condition in children not yet affected clinically. Since the level is raised in affected children, the possibility of using this estimation to detect heterozygous carriers of genes responsible for the various forms of the disease has been considered. The highest levels are seen in the severe sex-linked recessive forms early in the disease (including the stage at which clinical symptoms have not yet appeared). Measurement of LDH M4 isoenzyme helps determine the location and severity of the muscle damage. ${ }^{9}$ The levels may be used to monitor acute (as in the case of a traumatic injury) or chronic progressive conditions such as muscular dystrophy. ${ }^{10}$ Combined with muscle biopsy and electrophysiology, serum CPK activity is a valuable adjunct; more disease entities were clearly identified and diagnostic accuracy improved. ${ }^{11}$ All these criteria, by then refined by much study and scientific discourse, resulted in a classification that included the X-linked Duchenne (DMD) and Becker (Dystrophinopathies), LGMD (sarcoglycanopathies), congenital (laminopathies) and Emery-Dreifuss muscular dystrophy (EDMD), the autosomal dominant facioscapulohumeral (FSHD), distal and oculopharyngeal types, as well as the multisystem disorder, myotonic dystrophy. The names of many of these types clearly indicate the primary diagnostic importance of detailed recording of the distribution of affected muscles; the distinction between DMD and BMD, and between the CMD and LGMD types, depended on the timing and progression of symptoms. ${ }^{1}$ Indeed, it was recognized that at least one autosomal dominant form of LGMD existed, although its distinction from BMD or from the Duchenne carrier state, in which proximal muscular weakness sometimes occurs, was a particular problem, and serious errors in the consequent genetic advice were not uncommon. ${ }^{12,13}$ Furthermore, the use of CPK estimation in carriers of the Duchenne dystrophy introduced a useful (though fallible) technique for the scientific study of the carrier state and its genetic implications. ${ }^{14}$

As muscular dystrophies are relatively uncommon disorders, very little work has been done in Pakistan regarding their clinical classification. This study has provided (for the first time in Pakistan) the role of biochemical and clinical parameters for classifying different types of muscular dystrophies. 


\section{METHODOLOGY}

A total of 100 patients comprising of all age groups, both genders and with strong clinical suspicion of muscular dystrophy, with or without positive family history, were included through convenient, nonprobability sampling technique. These patients were visiting the Children Hospital Lahore and PSRD (Pakistan Society of Rehabilitation of Disabled) Hospital, 101, Ferozpur Road, Lahore which is the largest institute in Punjab dealing with and managing the patients with muscular dystrophies and other disabilities.

This observational, descriptive study was conducted in the Departments of Morbid Anatomy and Histopathology and Human Genetics and Molecular Biology at University of Health Sciences Lahore during three years' time (March, 2012-March 2015).

The clinical criterion of muscular dystrophies suggested by Pereira (Box-1) was adopted to recruit the patients. Patients with traumatic muscle weakness and/or wasting, with electromyography (EMG) and/or nerve conduction study (NCS) results depicting the neural/neuromuscular origin of the disease were excluded from the study. An informed written consent from the patient or a guardian/ parents of the patient fulfilling the above mentioned clinical criteria was taken. Detailed clinical history and physical examination findings were recorded in predesigned proformas.

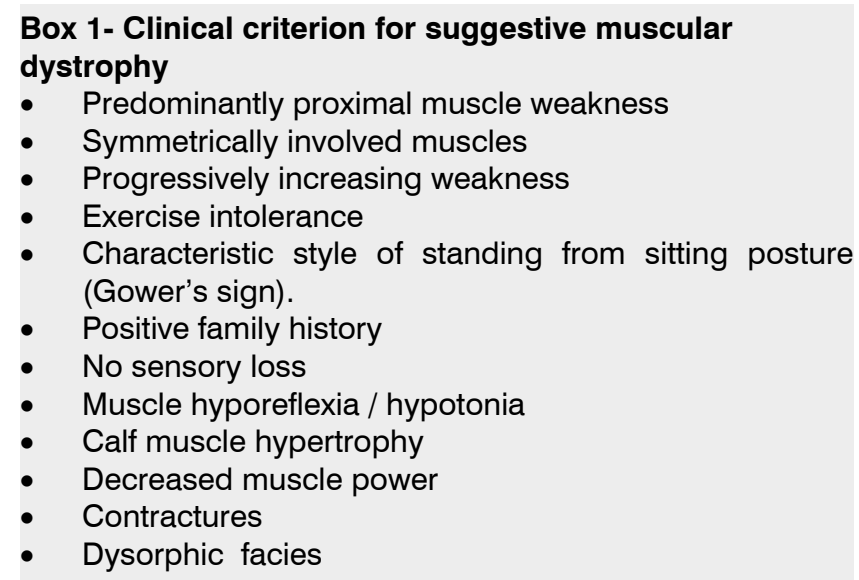

Forbiochemical investigations, blood wassampled by venipuncture, allowed to clot, centrifuged (3500 $\mathrm{rev} / \mathrm{min}$ for $5 \mathrm{mins})$. The supernatant serum was collected for determination of serum (i) Creatine Phosphokinase (CPK) levels according to the recommendations of German Society of Clinical Chemistry (ii) Lactate Dehyrogenase (LDH) (Biovision, USA) and (iii) Aldolase (RANDOX, UK) levels by manual method on Metrolab 1600 DR.

\section{RESULTS}

A total of 100 patients including $n=77$ males and $\mathrm{n}=23$ females were included. Mean age of the total 100 patients was $12.1990+6.69913$ years (Range $=3.5-36$ years).

All patients reported from different cities and districts from all over Punjab including Okara, Sahiwaal, Sheikhupura, Mureedke, Shadrah, Sargodha, Sialkot, Multan, Gujrat, Jarranwala, Bhawalpur, Bahawalnagar and Wazirabad while majority of patients $(46 \% ; 35 \%$ males, $11 \%$ females) belonged to Lahore.

The clinical features of these 100 patients are summarized in Table-I

Parents of $n=76$ patients reported with consanguineous marriages. As regards gender, consanguinity was more distributed among females $(82.6 \%$ vs $74 \%) \quad(p=0.002)$. A total of $\mathrm{n}=31$ patients presented with a positive maternal and paternal sided family history of muscular dystrophy in close relatives.

A total of $n=87$ cases including all males and $n=22$ $(95.7 \%)$ females presented with symmetrical muscle involvement (lower limb followed by upper limb). Involvement of the lower limbs as a primary weakness followed by symmetrical and severe involvement of the upper limb girdle and lower limb girdle suggested LGMD in 9 (6F,3M) patients. Involvement of shoulders and upper limbs as a primary weakness followed by facial muscles and infrequent involvement of lower limbs suggested FSHMD in 2 cases. Involvement of upper limbs as a primary weakness followed by lower limbs with a moderate rise in CPK levels and mild phenotype was seen in two female patient which was quite suggestive of dysferlinopathy. 


\section{Clinical Features}

Mean age at onset of weakness

Mean duration of muscle weakness

Mean age of ambulation loss

Fymptom

Delayed milestones

Ambulation

Exercise intolerance

Consanguinity

Family history

Socioeconomic status

Gower's sign

Calf muscle pseudo-hypertrophy

Primary muscles involved

Pattern of muscle involvement

Dysmorphic faces

Toe walking

Waddling gait

Reduced muscle power

Scapular winging

Perinatal complications

Spinal rigidity /Lumbar lordosis

Progressive joint contractures

Co-morbid conditions

Jaw muscle functions

\section{Values /Frequency}

$5.905 \pm 4.455$ years

$6.40 \pm 4.266$ years

$15.905 \pm 3.412$ years

Frequent falls

Difficulty in getting up from lying or sitting posture

Inability to walk

Waddling gait

Weakness of the upper limb muscles

Walking only

Walking and speech only

Partial loss

Complete loss

Normal

Mild

Moderate- severe

First Cousins

Second Cousins

Maternal

Paternal

Upper

Middle

Lower

Positive

Mild

Moderate

Absent

Lower limbs followed by upper limbs

Upper limbs followed by lower limbs

Upper limbs with shoulder girdle only

Lower limbs with pelvic girdle only

Symmetrical

Asymmetrical

Present

Present

Present

Lower limbs and upper limb both

Lower limbs only

Present

Present

Present

Ankle and knee joints both

Ankle joint

Cardio-pulmonary complications

Other

Reduced

Normal
$63 \%$

$17 \%$

$9 \%$

$7 \%$

$4 \%$

$15 \%$

$10 \%$

$82 \%$

$14 \%$

$4 \%$

$4 \%$

96\%

$65 \%$

$11 \%$

$18 \%$

$13 \%$

$3 \%$

$15 \%$

$82 \%$

94\%

$84 \%$

$14 \%$

$2 \%$

$89 \%$

$1 \%$

$7 \%$

$3 \%$

99\%

$1 \%$

$6 \%$

$11 \%$

$80 \%$

$62 \%$

$38 \%$

$4 \%$

$5 \%$

$6 \%$

$10 \%$

90\%

$18 \%$

$7 \%$

$2 \%$

98\%

Table-I. Clinical Features of $n=100$ Patients with Muscular Dystrophies 
A total of $n=82$ cases managed to ambulate with support of other persons while $n=14$ patients were totally non ambulatory. As regards the loss of ambulation, only two male patients reported onset of weakness and ambulation loss before 1 year of age that may represent CMD, dystroglycanopathy or congenital onset of LGMD (cLGMD).

Positive Gower's sign was demonstrated by $n=79$ cases who were partial ambulatory. All $n=14$ non ambulatory cases, who could not be tested for this sign, also reported positive Gower's sign in the past. These findings therefore confer that Gower's sign is a nonspecific but pathognomic clinical feature for all types of muscular dystrophies.

Six patients presented with dysmorphic faces including coarsening of facial features, widened epicanthic folds, protuberant upper jaw and high arched palate. Reduced jaw muscle movements including closing and/or retracting mandible (medial pterygoid, masseter / temporalis), opening, protruding and side-to- side movement of mandible (lateral pterygoid) were seen in $n=2$ patients. Chronic co-morbid conditions that were reported included respiratory insufficiency with or without chronic obstructive pulmonary diseases (COPD), cardiac complications, juvenile diabetes, recurrent upper respiratory and urinary tract infections, tuberculosis, jaundice, etc. Perinatal complications reported were hypotonia, high grade fever, low birth weight, jaundice etc.

On examination, ankle jerk was exaggerated in $n=$ 78 (16F, 62M) patients while other reflexes were normal in all patients. Nerve conduction velocity (NCV) demonstrated non-neural pathology while EMG diagnosed myopathy in all (100) cases.

Mean serum CPK level in $n=100$ patients was $2376.6364 \pm 910.78963 \mathrm{U} / \mathrm{L}$. Mean serum LDH and Aldolase levels in $n=100$ patients were $1030.7800 \pm 180.1620 \mathrm{U} / \mathrm{L}$ and $10.089 \pm 1.525$ $\mathrm{U} /$ L respectively. Pearson Chi Square test revealed that the patients showed significant association $(p<0.05)$ between duration of weakness, calf muscle hypertrophy, toe walking, waddling gait and contractures with the enzymes levels.

\section{DISCUSSION}

Duchenne muscular dystrophy, the most common form of muscular dystrophy in children, is an X-linked, severe and progressive muscular dystrophy with a birth prevalence of 1 in 3500 live born males. ${ }^{4,15}$ The milder Becker Muscular Dystrophy (BMD), a variant of DMD, shows a less severe but more variable phenotype, ranging from a slightly less severe DMD-like condition to a very mild condition. Overall, the physical picture of BMD patients resembles with that of DMD. The main difference with DMD is in the age of onset (later for BMD) and rate of progression. ${ }^{16,17}$ The prevalence of BMD at birth is 1 in 8500 live born males. In contrast to DMD they show more heterogeneous profile, clinical phenotypes and the morphological changes vary greatly. ${ }^{18}$

In the present study, mild-moderate phenotype that related significantly to serum CPK levels classified the patients as BMD based on their later age of onset. Rest 74 cases with moderatesevere phenotype and very high CPK levels were classified as of having DMD. The limb girdle muscular dystrophies are classically defined as muscular dystrophies that involve proximal upper and lower limbs muscles predominantly. Some features in LGMD which could be found in addition to typical proximal weakness include scapular winging, dysarthria, contractures, scoliosis and distal muscle involvement. In the present study, 9 patients $(6 \mathrm{~F}, 3 \mathrm{M})$ presented with the typical clinical pattern suggestive of LGMD. One male and 3 females were partial ambulatory with moderate phenotype and involvement of upper limb girdle. The other 3 female and 2 male patients were non ambulatory with severe phenotype and lower limb girdle involvement. Involvement of girdles, however, was observed not as a primary manifestation but following the significant motor weakness of the limbs. LGMD was first described as a clinical entity by Watson and Nattrass (1954). They are termed as such, as they share the characteristic features of muscle weakness predominantly affecting the shoulder and pelvic girdle. The LGMD are broadly divided 
into two i-e autosomal dominant (designated LGMD2) and autosomal recessive (LGMD1), and these two are now further subdivided into 19 subtypes on the basis of the clinical diversity and genetic mutations found in them ${ }^{2,19} \mathrm{~A}$ few of the recessive forms have been linked to the mutations in proteins that constitute the dystrophin-glycoprotein complex. ${ }^{20}$ Mutations in dysferlin, a protein that is thought to interact with Caveolin 3, cause LGMD2B. ${ }^{21,22}$ Dysferlin is the protein product of the 2 p13 gene and is normally localized to the muscle plasma membrane. Two female patients in the present study presented with predominant upper limb involvement with girdle sparing and milder phenotype with no respiratory and cardiac muscles involvement, which is consistent with the reports from other authors $^{22,23,24}$ and hence classified as a patient with dysferlinopathy.

Facioscapulohumeral muscular dystrophy is a progressive degenerative disease that initially affects the muscles of the face, shoulders, and upper arms followed by the muscles of the feet, pelvic girdle, and abdomen. Affected individuals may also suffer from hearing loss. Onset and progression of the disease is variable and often the weakness is asymmetrical in affected individuals. ${ }^{25}$ The essential clinical features are weakness of facial muscles, scapulohumeral, tibialis anterior and pelvic girdle muscle involvement which was the feature seen in the present study in one male patient, 11 years of age having moderate to severe phenotype. Many patients are only mildly affected as the other patient, 27 years male, diagnosed of having FSHMD in the present study. ${ }^{26}$ CMDs associated with the deficiency of Laminin alpha-2 and collagen- $\mathrm{VI}$ are the commonest subtypes in many countries especially of European origin. ${ }^{27}$ Congenital muscular dystrophy is comparatively infrequent autosomal recessive form of dystrophy existing at birth or in initial infancy with hypotonia, generalized weakness and contractures as the presenting symptoms. With only authentic report of 0.68 to 2.5 per 100,000 point prevalence by Peat et al (2008)..$^{28}$ Inability to diagnosis of primary muscle weakness with and without intellectual debility may have led to a continued underestimation of its prevalence. ${ }^{29,30}$ Furthermore, the comparative frequency of CMD subtypes differs in different populations. ${ }^{31}$ The important differential diagnosis in this context is the cLGMD that can have its onset at childhood, even if symptoms appear more frequently in adolescence or young adulthood. ${ }^{20}$ The present study reports one male patient, 3 years of age with onset of weakness at 6 months, who presented with a progressive muscle weakness leading to a moderate phenotype with dysmorphic facies, jaw weakness and COPD at the time of presentation. Cranial MRI suggested white matter changes. The other male patient, 5 years of age with onset of limb weakness in perinatal period, presented with a severe phenotype including jaw weakness and associated COPD. No mass lesion on CT Scan or evidence of intellectual impairment was seen which was consistent with the findings reported by Mercuri et al. ${ }^{30}$

Since early $20^{\text {th }}$ century, the diagnosis of muscle dystrophies is aided by the very significant laboratory assistance provided by serum CPK estimations, an invaluable test pioneered by Hideo Sugita and Setsuro Ebashi. ${ }^{32}$ Warburg and Christian (1943) reported incidental finding of raised serum aldolase in case of muscular dystrophies. Later on it was also confirmed by Sibley and Fleisher (1954) who later on also found that the enzyme was not raised in other neuromuscular diseases. ${ }^{33,34}$ The patients in the present study also demonstrated at least 10-150 times raised enzyme levels that related significantly with the severity of disease.

Females may be the obligate carriers i-e a woman with at least one affected son and one affected male member or with two or more affected sons. They can be possible carriers i-e mothers of isolated case of muscular dystrophy, would be a manifesting/ symptomatic carrier when she is a female with positive family history in males, raised serum creatine phosphokinase (CPK) and some proximal muscle weakness. ${ }^{7}$ In present study, 4 of 23 female patients presented with very milde phenotype with mildly raised CPK levels that depicted carrier status in them. 


\section{CONCLUSION}

Muscular dystrophies are not only restricted to dystrophinopathies in our population. Studying patients with a wide range of clinical phenotypes in the present study, aided not only in approaching the clinical diagnosis of different types of muscular dystrophies but also raises the need for detection of abnormal proteins of the DAG complex by employing appropriate histological and/or gene mutation analysis for precise classification.

\section{Copyright(C) 28 July, 2016.}

\section{REFERENCES}

1. Hassan MJ, Mahmood S, Ali G, Bibi N, Waheed I. Intragenic deletions in the DMD gene in 211 Pakistani Duchene muscular dystrophy patients. Pediat Internat, 2008; 50: 162-6.

2. Walton JN and Nattrass FJ. On the classification, natural history and treatment of the myopathies. Brain, 1954; 77: 169.

3. Ozawa E, Nishino I, Nonaka I. Sarcolemmopathy: muscular dystrophies with cell membrane defects. Brain Pathology, 2001; 11: 218-230.

4. Guang-Qian Z, Hui-Qi X, Su-Zhen Z, Zhi-Ming Y. Current understanding of dystrophin-related muscular dystrophy and therapeutic challenges ahead. China Med J, 2006; 119: 1381-1391.

5. Kaspar RW, Allen HD, Montanaro F. Current understanding and management of dilated cardiomyopathy in Duchenne and Becker muscular dystrophy. J Americ Acad Nurse Pract, 2009; 21 (5): 241-249.

6. Engel AG, Yamamoto $\mathrm{M}$, Fischbeck $\mathrm{KH}$. Dystrophinopathies. In: Engel, A.G., FranziniArmstrong, C.Z. eds. Myology. McGraw-Hill Inc, New York, 1994: pp 1133-1187.

7. Freund AA, Scola $\mathrm{RH}$, Arndt RC, Lorenzoni PJ, Kay CK. Duchenne and Becker muscular dystrophy: A molecular and immunohistochemical approach. Arq Neuro-Psiqiatr, 2007; 65:73-76.

8. Wolf PL. History of diagnostic enzymology: A review of significant investigations. Clinica Chimica Acta, 2006; 369: 144-147.

9. Pincus MR, Abraham NZ Jr, Carty RP. Clinical enzymology. In: McPherson RA, Pincus MR, eds. Henry's Clinical Diagnosis and Management by Laboratory Methods. 22nd ed. Philadelphia, PA: Elsevier Saunders; 2011: chap 20.
10. Yamini D. Kids Health, Blood Test: Lactate Dehydrogenase (LDH), 2014. Available online at http:// kidshealth.org/parent/system/medical/test_Idh.html\#.

11. Zatz M. and Otto PA. Evaluation of carrier detection rate for Duchenne and Becker muscular dystrophies using serum creatine-kinase (CK) and pyruvate kinase (PK) through discriminant analysis. Am J Med Genet, 1986; 25, 219-231.

12. Lai KKS, Lo IF, Tong TMF, Cheng LYL, Lam STS. Detecting exon deletions and duplications of the DMD gene using Multiplex Ligation-dependent Probe Amplification (MLPA). Clinic Biochem, 2006; 39: 367-372.

13. Darras BT, Miller DT, Urion DK. Dystrophinopathies. 2000 Sep 5 [Updated 2014 Nov 26]. In: Pagon RA, Adam MP, Ardinger $\mathrm{HH}$, et al., editors. Gene Reviews ${ }^{\circledR}$ [Internet]. Seattle (WA): University of Washington, Seattle; 1993-2016. Available from: http://www.ncbi. nlm.nih.gov/books/NBK1119/.

14. Hugh J, McMillan MG, Basil TD, Peter B. Serum Transaminase Levels in Boys with Duchene and Becker Muscular Dystrophy. Pediatrics, 2011; 127: 132-6.

15. Shukla G, Bhatia C, Sarkar C, Padma MV, Tripathi $M$ and Jain $S$. Muscular dystrophies and related skeletal muscle disorders in an Indian population-a prospective correlative study. J Clin Neurosci, 2004; 11:723-727.

16. Gurvich OL, Tuohy TM, Howard MT. DMD pseudo exon mutations: splicing efficiency, phenotype, and potential therapy. Ann Neurol, 63: 2008; 81-9.

17. Ashton EJ, Yau SC, Deans ZC. Simultaneous mutation scanning for gross deletions, duplications and point mutations in the DMD gene. Eur. J. Hum Genet, 2008; 16:53-61.

18. Blake DJ, Weir A, Newey SE, Davies KE. Function and genetics of dystrophin and dystrophin-related proteins in muscle. Physiol Rev, 82: 2002; 291 - 96.

19. Victor D, Caroline SA, Russell LJM. Muscle biopsy. Philadelphia: Saunders Elsevier, $3^{\text {rd }}$ ed. USA. 2007.

20. Norwood F, Visser M, Eymard B, Lochmuller H, Bushby $K$. EFNS guideline on diagnosis and management of limb girdle muscular dystrophies. Eur J Neurol, 14: 2007; 1305-12.

21. Bisceglia L, Zoccolella S, Toracco A, Piemontese MR, Dell'Aglio R, Amati A, De Bonis P, Artuso L, Copetti M, Santorelli FM, Serlenga L, Zelante L, Bertini E, Petruzzella V. A new locus on 3p23-p25 for an 
autosomal dominant limb-girdle muscular dystrophy, LGMD1H. Europ J Hum Genet, 2010; 18: 636-41.

22. Bansal D, Miyake $K$, Vogal SS. Defective membrane repair in dystrophin-deficient muscular dystrophy. Nature, 2003;3:168-72.

23. Han R, Campbell KP. Dysferlin and muscle membrane repair. Curr Opin Cell Biol, 2007; 19:409-16.

24. Nagashima T, Chuma T, Mano Y, Goto Y, Hayashi YK, Minami N, Nishino I, Nonaka I, Takahashi T, Sawa H, Aoki M, Nagashima K. Dysferlinopathy associated with rigid spine syndrome. Neuropathology, 2004; 24:341-6.

25. Maarel VSM, Frants RR, Padberg GW. Facioscapulohumeral muscular dystrophy. Biochim Biophys Acta, 2007; 1772(2):186-94.

26. Sacconi S, Camano P, de Greef JC, Lemmers RJLF, Salviati L, Boileau P, Lopez de Munain AA, van der Maarel SM, Desnuelle C. Patients with a phenotype consistent with facioscapulohumeral muscular dystrophy display genetic and epigenetic heterogeneity. J Med Genet, 2012; 49: 41-46.

27. Sparks SE and Escolar DM. Congenital muscular dystrophies. Handb Clin Neurol, 2011; 101: 47-79.

28. Peat RA, Smith JM, Compton AG, Baker NL, Pace
RA, Burkin DJ, Kaufman SJ, Lamande SR, North KN. Diagnosis and etiology of congenital muscular dystrophy. Neurology, 2008; 71:312-21.

29. Norwood FL, Harling C, Chinnery PF, Eagle M, Bushby $\mathrm{K}$, Straub V. Prevalence of genetic muscle disease in Northern England: in-depth analysis of a muscle clinic population. Brain, 2009; 132:3175-86.

30. Mercuri, E, Messina S, Bruno C, Mora M, Pegoraro E, Comi GP, D’Amico A, Aiello C. et.al. Congenital muscular dystrophies with defective glycosylation of dystroglycan: a population study. Neurology, 2009; 72:1802-9.

31. Okada M, Kawahara G, Noguchi S, Sugie K, Murayama K, Nonaka I, Hayashi YK, Nishino I. Primary collagen VI deficiency is the second most common congenital muscular dystrophy in Japan. Neurology, 2007; 69:1035-42.

32. Ebashi S, Toyokura Y, Momoi H, Sugita H. High creatine phosphokinase activity of sera of progressive muscular dystrophy. J Biochem Tokyo, 1959; 46: 103104.

33. Warburg O, Christian W, Taylor JF. Biochem. Z., 1943; 314.

34. Sibley JA. Significance of serum aldolase levels. Ann N Y Acad Sci. 1958; 75(1):339-348.

\section{AUTHORSHIP AND CONTRIBUTION DECLARATION}

\begin{tabular}{|c|l|l|}
\hline Sr. \# & \multicolumn{1}{|c|}{ Author-s Full Name } & \multicolumn{1}{|c|}{ Contribution to the paper } \\
\hline 1 & Dr. Nadia Naseem & A, B, C, D \\
2 & Dr. Saqib Mahmood & B, D \\
3 & Dr. Afzal Hussain & A \\
4 & Dr. Mumtaz Hussain & A Signature \\
5 & Miss Ayesha Munir & A \\
6 & Mr. Waqas Latif & A \\
7 & Dr. A.H. Nagi & A, C, D
\end{tabular}

A. Substantial contribution to conception or design of the work; or the acquisition, analysis or interpretaion of data for the work.

B. Drafting of the work or revising it critically for important intellectual content.

C. Final approval of the version to be puslished.

B. Agreement to be accountable for all aspects of the work in ensuring that questions related to the accuracy or integrity of any part of the work and appropriately investigated and resolved. 